\title{
Elevated Serum Lipase Levels as a Marker of Intraductal Papillary Mucinous Neoplasm of the Pancreas: A Cross-Sectional Study
}

Rieko Okada ( $\square$ rieokada@med.nagoya-u.ac.jp)

Nagoya University Graduate School of Medicine https://orcid.org/0000-0002-6206-2501

Takashi Okada

Okada Medical Clinic

Akira Okada

Okada Medical Clinic

Kenji Wakai

Nagoya University Graduate School of Medicine

Research article

Keywords: Intraductal papillary mucinous neoplasms of the pancreas, Pancreatic neoplasms, Hyperlipasemia, Lipase, Magnetic resonance cholangio-pancreatography

Posted Date: September 24th, 2020

DOI: https://doi.org/10.21203/rs.3.rs-72362/v1

License: (9) This work is licensed under a Creative Commons Attribution 4.0 International License. Read Full License 


\section{Abstract}

Background: Intraductal papillary mucinous neoplasm (IPMN) is a well-established premalignant pancreatic lesion. It is known that mild hyperlipasemia, elevated serum lipase levels, is the risk of malignancy and poor prognosis in patients with IPMN. The purpose of this study is to reveal whether hyperlipasemia is useful in identifying patients with IPMN.

Methods: The consecutive 91 patients aged 40-89 years who underwent magnetic resonance cholangiopancreatography (MRCP) from 2016 to 2019 and who showed elevated serum lipase values above the reference range $(>51 \mathrm{U} / \mathrm{L})$ in one year were enrolled. Patients were categorized into three as having IPMN, other pancreatic cystic lesion, and non-cysts groups by MRCP.

Results: IPMN was found in $22 \%$ and other pancreatic cystic lesion was found in $17 \%$ of patients. The prevalence of IPMN was higher in patients with mild hyperlipasemia $(60-79 \mathrm{U} / \mathrm{L})$ compared to those with borderline hyperlipasemia (<60 U/L) (36\% vs. 17\%; odds ratio, 5.2; $95 \%$ confidence interval, 1.2-22.4). Patients taking $\mathrm{H} 2$-blockers or weight loss $>1 \mathrm{~kg} /$ year also showed higher risk of IPMN (39\% vs. $15 \%$ and $32 \%$ vs. $19 \%$, respectively), and patients with both mild hyperlipasemia and taking $\mathrm{H} 2$-blockers showed the highest risk of IPMN compared to those with neither of them (75\% vs. $13 \%)$.

Conclusions: Patients with mild hyperlipasemia showed five times higher risk of having IPMN compared to those with borderline hyperlipasemia. Further investigation with MRCP should be considered for patients with mild hyperlipasemia, especially when they had episodes of epigastric discomfort or weight loss, to find high risk patients for future pancreatic carcinoma.

\section{Background}

Intraductal papillary mucinous neoplasm (IPMN) is a well-established premalignant pancreatic lesion with a stepwise progression from low-grade dysplasia to high-grade dysplasia and finally invasive carcinoma [1-3]. IPMN is characterized by the papillary growth of the ductal epithelium with rich mucin production, which is responsible for cystic segmental of diffuse dilatation of the main pancreatic duct (MPD) and/or its branches [1]. The mean frequency of malignancy for main-duct IPMN is about $60 \%$, while that for branch-duct IPMN is about $25 \%$ [1, 2], so IPMN is the main indication for pancreatic resection [4]. If it is discovered in the initial stage, they have a good prognosis. Thus, IPMN represents a potential "window of opportunity" for pancreatic cancer prevention and early detection [4].

To date, there is no serum marker to predict the presence of IPMN which can be used for screening $[1,2]$. Serum lipase is a marker of inflammation of the pancreas and also is a marker of pancreatic cancer $[5,6]$. Former studies showed that mild hyperlipasemia (elevated serum lipase values above $60 \mathrm{U} / \mathrm{L}$ ) showed 3-4 times higher risk of invasive IPMN [4, 7] and poorer prognosis (survival rate was only $60 \%$ after 2 years) [8] in patients with IPMN. Recent reviews mention that asymptomatic hyperlipasemia may occur in some (about $5 \%$ ) of the patients with IPMN $[1,2,9,10]$, but the supporting data is scarce. Among patients with hyperlipasemia but negative findings on ultrasonography, $7 \%$ of patients developed IPMN 
after three years $[11,12]$. However, no report has shown the prevalence of IPMN in patients with hyperlipasemia. As temporary pancreatic duct obstruction secondary to abundant mucus secretion may occur in patients with IPMN [13], we hypothesized that hyperlipasemia can be a marker of IPMN.

The purpose of this study is to reveal whether hyperlipasemia, elevated serum lipase levels, is useful in identifying patients with IPMN.

\section{Methods}

\section{Study subjects}

The consecutive patients who underwent magnetic resonance cholangio-pancreatography (MRCP) from January 2016 to December 2019 at Okada Medical Clinic in Nagoya, Japan, were enrolled $(n=120)$. Data were collected during routine care and were extracted from medical records retrospectively. Subjects without data on serum lipase were excluded $(n=6)$. Among them, patients who showed elevated lipase value above the reference range $(>51 \mathrm{U} / \mathrm{L})[4,14]$ at least once during one year prior to MRCP were included, remaining 91 patients (38 male and 53 female, aged 40-89 years). Patients without any elevation in lipase were excluded from the analysis, because those patients underwent MRCP for other reasons (e.g. follow-up for known liver or kidney diseases). Patients were checked whether they had any explainable non-pancreatic causes for their lipase elevation, such as gallstones or renal insufficiency.

This study was approved by the Ethical Committee of Nagoya University Graduate School of Medicine (approval number 2020-0075) and the ethics committee of Nagoya Medical Association.

\section{Serum lipase values and hyperlipasemia}

Serum lipase values were checked for patients with unknown cause of dyspepsia to check the underlying pancreatic disorder as described elsewhere [15]. Some patients with clinical symptoms such as epigastric discomfort, back pain, and weight loss, or patients with worsened or newly diagnosed diabetes mellitus were also checked for their serum lipase values [6]. Then, patients who showed both elevated lipase values above the reference range (>51 U/L) [4] and prior clinical symptoms were considered to undergo MRCP to check the possible pancreatic neoplasms as recommended in the Clinical Practice Guidelines for Pancreatic Cancer 2019 from the Japan Pancreas Society [6].

Serum lipase values were determined by the DGGMR method (Signas Auto LIP; Shino-Test Corp., Tokyo, Japan). The latest lipase value within 1 month before MRCP was used. Patients were categorized into three groups by the serum lipase levels $(<60,60-79$, and $\geq 80 \mathrm{U} / \mathrm{L}$ for borderline, mild, and moderate hyperlipasemia, respectively) according to the former studies $[7,8,12,16]$.

\section{IPMN and other pancreatic cystic lesion assessed by MRCP}

MRCP/MRI was taken at Nagoya Clinical Examination Center of Nagoya Medical Cooperative Association (1.5T MRI, Siemens) and reported by the certified radiologists at this center. Each report of 
MRCP in the medical record was retrospectively checked. Patients were categorized into three as having IPMN, cysts, and non-cysts groups. Branch-duct IPMN was defined by reports of certified radiologists that pancreatic cysts of $>5 \mathrm{~mm}$ in diameter that communicate with the MPD as defined in the Fukuoka Guideline. ${ }^{3}$ No main-duct IPMN or mixed-type IPMN was diagnosed. Patients with pancreatic cystic lesion other than IPMN were categorized into "cysts" group. IPMN and cysts were checked for their location (head, body, or tail of the pancreas), size of the largest cyst, and multifocality. The presence of MPD dilatation, pancreatic degeneration, liver cysts, renal cysts, gallstones, and adenomyomatosis of gallbladder were also checked from the radiologists' report.

\section{Other measurements}

All the information was taken from the medical record retrospectively. Data about systolic/diastolic blood pressure, weight, height, urine protein, blood tests including alkaline phosphatase and gamma-glutamyl transpeptidase, and medications were obtained from the medical record. Histamine $\mathrm{H} 2$-receptor antagonists (H2-blockers), proton pump inhibitors (PPI), or camostat mesilate were prescribed for patients with clinical symptoms such as epigastric discomfort. Patients' weight one year prior to MRCP examination was also obtained, and weight change was calculated. The glomerular filtration rate (eGFR) was estimated using the Japanese eGFR equation [17].

Present symptoms, past history, family history, and drinking and smoking habits were asked by the trained nurses or doctors at the time of MRCP. Present symptoms including epigastralgia, back pain, nausea, diarrhea, and appetite loss, history of acute/chronic pancreatitis, and parents' history of pancreatic carcinoma were asked according to the former studies $[1,10]$. Former and current smoking and drinking habits were asked, and the amounts of daily alcohol were asked.

Each report of gastroscopy within 6 months prior to MRCP was checked for the presence of gastric ulcer or cancer and positivity of Helicobacter pylori.

\section{Statistical analyses}

Characteristics of study patients were compared between the three groups (IPMN, cysts, and non-cysts groups). The prevalence of IPMN and cysts according to the serum lipase values were shown $(<50,50-$ $59,60-69,70-79$, and $\geq 80 \mathrm{U} / \mathrm{L})$.

Then, the prevalence of IPMN and cysts were compared according to the serum lipase levels $(<60,60-79$, and $\geq 80 \mathrm{U} / \mathrm{L}$ for borderline, mild, and moderate hyperlipasemia, respectively). Odds ratios (OR) for the prevalence of IPMN and cysts were estimated using unconditional logistic regression models with $95 \%$ confidence intervals $(\mathrm{Cl})$. ORs were adjusted for age groups (40-49, 50-59, 60-69, 70-79, and 8089 years old), sex, and eGFR levels ( $<45,45-59,60-89$, and $\left.\geq 90 \mathrm{~mL} / \mathrm{min} / 1.73 \mathrm{~m}^{2}\right)$ [18], because serum lipase values are affected by kidney function $[19,20]$.

Finally, characteristics of IPMN and other cysts were compared for their location, size, multifocality, and coexisting findings on MRCP. STATA version 9 (STATA Corp., College Station, Tex., USA) was used for 
these analyses.

\section{Results}

Characteristics of IPMN, cysts, and non-cysts groups were shown in Table 1. Patients with IPMN are more likely to be older male, losing their weights, and taking H2-blockers. There were no differences in the prevalence of present symptoms, history of chronic pancreatitis, ever smokers and drinkers between the groups. No patients suffered acute pancreatitis in the past. More than half of the patients undertook gastroscopy, and no gastric ulcer or cancer was found. Positive rate of Helicobacter pylori was not different between the groups. Most of the symptoms were relieved after taking $\mathrm{H} 2$-blockers, PPI, or camostat mesilate.

In total, IPMN was found in $22 \%$ of patients who showed elevated lipase values above the reference range (> $51 \mathrm{U} / \mathrm{L})$ at least once during one year. The prevalence of IPMN increased with increasing values of the latest serum lipase $(13 \%, 19 \%, 25 \%$, and $63 \%$ for patients with lipase values of < 50, 50-59, 60-69, and 70-79 U/L, respectively). However, patients with elevated lipase values above $80 \mathrm{U} / \mathrm{L}$ did not show increased prevalence of IPMN (14\%) [Figure 1].

Overall, the prevalence of IPMN was higher in patients with mild hyperlipasemia (60-79 U/L) compared to those with borderline hyperlipasemia (< $60 \mathrm{U} / \mathrm{L})$ (36\% vs. 17\%; adjusted OR, 5.2; 95\% Cl, 1.2-22.4 for patients with mild hyperlipasemia compared to those with borderline hyperlipasemia) [Table 2]. The prevalence of cysts tend to be increased with increasing levels of serum lipase (9\%, 18\%, and $25 \%$ for patients with lipase level of $<60,60-79$, and $\geq 80 \mathrm{U} / \mathrm{L}$, respectively), although the association was not statistically significant. Patients who had any explainable causes for their lipase elevation except IPMN or pancreatic cysts (e.g. gallstones or renal insufficiency) were $49 \%, 29 \%$, and $54 \%$ for patients with lipase level of $<60,60-79$, and $\geq 80 \mathrm{U} / \mathrm{L}$, respectively.

Patients taking H2-blockers showed higher risk of IPMN (39\% vs. 15\%), and patients with weight loss $\geq$ $1 \mathrm{~kg}$ /year also showed higher risk of IPMN (32\% vs. 19\%). Patients with both mild hyperlipasemia (60$79 \mathrm{U} / \mathrm{L}$ ) and taking H2-blockers showed the highest risk of IPMN compared to those with neither of them (75\% vs. 13\%; OR, 21.2; 95\% Cl, 2.2-204.7 for patients with both mild hyperlipasemia and taking $\mathrm{H} 2-$ blockers compared to those with neither of them) [Table 2].

Finally, the characteristics of IPMN and cysts assessed by MRCP were compared in Table 3. The head of the pancreas was most frequently affected by IPMN (head 65\%, body 25\%, and tail 40\%), whereas cysts equally affected through pancreas. The size and multifocality was greater in IPMN than in cysts (The median size of the largest tumor/cyst, $9 \mathrm{~mm}$ vs. $3 \mathrm{~mm}$; multifocality, 45\% vs. 27\%). Liver cysts were more frequent in IPMN than in other cysts or in non-cysts groups (50\%, 7\%, and 32\% for IPMN, other cysts, and non-cysts groups, respectively), whereas the prevalence of renal cysts was not different between the groups. 


\section{Discussion}

To the best of our knowledge, we demonstrated for the first time that patients with mild hyperlipasemia (60-79 U/L) showed five times higher risk of having IPMN compared to those with borderline hyperlipasemia. Moreover, patients with mild hyperlipasemia who need to take H2-blockers showed the highest risk of having IPMN. Therefore, further investigation with MRCP should be considered for patients with mild hyperlipasemia, especially when they had episodes of epigastric discomfort, to find high risk patients for future pancreatic carcinoma.

The prevalence of IPMN is rarely reported. IPMN was found in $6.6 \%$ who underwent MRI or CT [21, 22]. Our study showed higher prevalence of IPMN (22\%), probably because all of our patients showed elevated lipase values at least once during one year. Moreover, the proportion of IPMN among all the pancreatic cystic lesions (IPMN and cysts) was greatly higher in our study (57\%) than previously reported (31-35\%) [23]. Thus, our study indicates that hyperlipasemia is useful in identifying patients with IPMN.

We found for the first time that patients with mild hyperlipasemia showed higher prevalence of IPMN (36\%) compared to those with borderline hyperlipasemia (17\%). One possible mechanism is that IPMN might cause subclinical pancreatitis. Subclinical pancreatitis has been described in patients with isolated increases in serum lipase/amylase levels but with no symptoms or imaging features [4]. IPMN is a wellknown etiology for acute pancreatitis [13,24-26], which occurs in about $12-67 \%$ of IPMN. Pancreatitis might be caused by hypersecretion of mucin and temporary obstruction of the pancreatic duct in IPMN, which causes elevation of pancreatic enzymes. Acute pancreatitis from IPMN might be unrecognized because typically acute pancreatitis from IPMN is predominantly mild and recurrent, with a long-standing asymptomatic hyperlipasemia [26, 27]. Thus, serum lipase is a better indicator of pancreatic inflammation than clinically defined pancreatitis [4], and we hypothesize that patients with IPMN may suffer subclinical inflammation of pancreas which causes hidden increase of serum lipase level, although episodes of acute pancreatitis were unrecognized in the past.

Another possible mechanism is that functional pancreatic disorder should be underlined. Functional pancreatic disorder is characterized by recurrent episodes of epigastric discomfort with elevated lipase/amylase without any causes of pancreatitis (e.g. alcohol, gallstones) [28, 29]. We formerly demonstrated that patients with unknown cause of dyspepsia had hyperlipasemia, which indicates that patients with unknown cause of dyspepsia may suffer from mild functional pancreatic disorders [15]. Associated symptoms of IPMN were epigastric discomfort (50-70\%) and weight loss (20-40\%) in the previous reviews $[1,10]$. Our report was consistent with these reviews that $55 \%$ of IPMN patients were taking medications for epigastric discomfort (H2-blckers, PPI, or camostat mesilate) and 30\% of IPMN patients showed weight loss, which were higher than those without IPMN. Patients with weight loss or who need to take H2-blockers might have higher risk of IPMN.

Moderate hyperlipasemia above $80 \mathrm{U} / \mathrm{L}$ did not show any increased risk of IPMN in our study. Hyperlipasemia is known to be associated with non-pancreatic disorders such as inflammation or obstruction of biliary tract or gastrointestinal tract, and renal insufficiency $[19,20]$. Interestingly, more 
than half of the patients (54\%) with moderate hyperlipasemia had some causes which can explain their increased lipase values (e.g. gallstones), whereas less than one-third of patients (29\%) with mild hyperlipasemia had any causes. As higher the lipase, the more patients had explainable causes for their lipase elevation, which may explain our findings. Thus, patients without any explainable causes of elevation in lipase should be monitored carefully.

Men and women were equally affected, elderly subjects aged 70th were frequently affected, and many of the cases were multifocal and located in the head of the pancreas in our study, which was consistent with the former studies [1-3]. Smoking and drinking were not associated with the risk of IPMN. The new finding is that liver cysts frequently coexist with IPMN in our study. Previous report showed that extra pancreatic cysts were frequent in patients with pancreatic cystic lesions [30], which may explain our result.

It is unknown whether patients with hyperlipasemia should undergo imaging examinations such as MRCP to detect pancreatic abnormalities. A former study showed that only $15 \%$ of patients with hyperlipasemia had pancreatic diseases [31], whereas another study showed half of the patients had pancreatic findings by MRCP $[32,33]$. Our study showed that one-third of the patients had IPMN and more than half of the patients had pancreatic cystic lesions among patients with mild hyperlipasemia. Thus, patients with hyperlipasemia should be assessed in order to diagnose pancreatic diseases [16, 33]. Furthermore, there should be a debate whether IPMN should have been found in asymptomatic stage, as asymptomatic patients had a lower risk of progression to malignancy [34]. It is known, however, that hyperlipasemia in patients with IPMN increase the risk of malignancy and showed poor prognosis $[4,7$, 8]. Thus, our patients should have high risk of malignancy of the pancreas. Further study is warranted to clarify whether IPMN with hyperlipasemia should be diagnosed in asymptomatic stage for the better prognosis.

Several limitations should be mentioned. First, the number of patients was rather small to show whether hyperlipasemia is a marker of IPMN. However, there is no data at all to show the prevalence of IPMN in patients with hyperlipasemia, and this is the first study to show that one-third of patients with mild hyperlipasemia had IPMN. Further study with larger number of patients is needed. Second, we could not show the increasing risk of IPMN by increasing level of serum lipase. This might be explained by that greater lipase level can be explained by non-pancreatic disorders such as gallstones, which should be confirmed in another study.

\section{Conclusions}

In conclusion, patients with mild hyperlipasemia, elevated serum lipase levels, showed five times higher risk of having IPMN compared to those with borderline hyperlipasemia. Patients with both mild hyperlipasemia and taking $\mathrm{H}$ 2-blockers or weight loss showed the highest risk of having IPMN. Thus, we suggest that patients with mild hyperlipasemia, especially when they had episodes of epigastric discomfort or weight loss, should be considered to undertake the imaging examination such as MRCP to 
detect pancreatic neoplasms including IPMN to find subsequent early stage of pancreatic cancer progression.

\section{Abbreviations}

IPMN: intraductal papillary mucinous neoplasm; MRCP: magnetic resonance cholangio-pancreatography; MPD: main pancreatic duct; PPI: proton pump inhibitors; eGFR: estimated glomerular filtration rate; OR: odds ratios; $\mathrm{Cl}$ : confidence intervals

\section{Declarations}

\section{Ethics approval and consent to participate}

The study is the retrospective observational research using only existing materials and not using human biological specimens. Therefore, obtaining patient consent is not deemed necessary according to the Japan's Ethical Guidelines for Epidemiological Research. The study was approved by the Ethical Committee of Nagoya University Graduate School of Medicine (approval number 2020-0075) and the ethics committee of Nagoya Medical Association.

\section{Consent for publication}

Not applicable

\section{Availability of data and materials}

The datasets used and/or analyzed during the current study are available from the corresponding author on reasonable request.

\section{Competing interests}

The authors declare that they have no competing interests.

\section{Funding}

None

\section{Authors' contributions}

RO analyzed data and mainly drafted the article, TO and AO contributed to the conception and design of the study, and KW revised the article critically for important intellectual content. All the authors approved the final version of the article to be published.

\section{Acknowledgements}


We appreciate Dr. Kenji Tanehiro and Drs. of Nagoya Clinical Examination Center of Nagoya Medical Cooperative Association for their contribution. We appreciate Ms. Kumi Seto, Ms. Emiko Fujita, Ms. Akiyo Shimono, Ms. Aiko Seko, Ms. Anna Koizumi, Ms. Haruka Baba, and all the staffs of Okada Medical Clinic for data collection.

\section{References}

1. Pagliari D, Saviano A, Serricchio ML, Dal Lago AA, Brizi MG, Lanza F, et al. Uptodate in the assessment and management of intraductal papillary mucinous neoplasms of the pancreas. Eur Rev Med Pharmacol Sci. 2017;21(12):2858-74.

2. Brugge WR. Diagnosis and management of cystic lesions of the pancreas. J Gastrointest Oncol 2015;6(4):375-88.

3. Tanaka M, Fernández-Del Castillo C, Kamisawa T, Jang JY, Levy P, Ohtsuka T, et al. Revisions of international consensus Fukuoka guidelines for the management of IPMN of the pancreas. Pancreatology. 2017;17(5):738-53.

4. Roch AM, Parikh JA, Al-Haddad MA, DeWitt JM, Ceppa EP, House MG, et al. Abnormal serum pancreatic enzymes, but not pancreatitis, are associated with an increased risk of malignancy in patients with intraductal papillary mucinous neoplasms. Surgery. 2014;156(4):923-9.

5. Sanh N, Fadul H, Hussein N, Lyn-Cook BD, Hammons G, Ramos-Cardona XE, et al. Proteomics Profiling of Pancreatic Cancer and Pancreatitis for Biomarkers Discovery. J Cell Sci Ther. 2018;9(4).pii:287.

6. Okusaka T, Nakamura M, Yoshida M, Kitano M, Uesaka K, Ito Y, et al; Committee for Revision of Clinical Guidelines for Pancreatic Cancer of the Japan Pancreas Society. Clinical Practice Guidelines for Pancreatic Cancer 2019 From the Japan Pancreas Society: A Synopsis. Pancreas. 2020;49(3):326-35.

7. Yagi Y, Masuda A, Zen Y, Takenaka M, Toyama H, Sofue K, et al. Predictive value of low serum pancreatic enzymes in invasive intraductal papillary mucinous neoplasms. Pancreatology. 2016;16(5):893-9.

8. Marsoner K, Haybaeck J, Csengeri D, Waha JE, Schagerl J, Langeder R, et al. Pancreatic resection for intraductal papillary mucinous neoplasm- a thirteen-year single center experience. BMC Cancer. 2016;16:844.

9. Pezzilli R, Calculli L. Branch-type intraductal papillary mucinous neoplasm of the pancreas: clinically and patient-reported outcomes. Pancreas. 2015;44(2):221-6.

10. Tanaka M, Kobayashi K, Mizumoto K, Yamaguchi K. Clinical aspects of intraductal papillary mucinous neoplasm of the pancreas. J Gastroenterol. 2005;40(7):669-75.

11. Pezzilli R, Morselli-Labate AM, Casadei R, Campana D, Rega D, Santini D, et al. Chronic asymptomatic pancreatic hyperenzymemia is a benign condition in only half of the cases: a prospective study. Scand J Gastroenterol. 2009;44(7):888-93. 
12. Antonini F, Belfiori V, Pagano N, Buscarini E, De Minicis S, Lo Cascio M, et al. Endoscopic Ultrasonography May Select Subjects Having Asymptomatic Chronic Pancreatic Hyperenzymemia Who Require a Stricter Follow-up. Pancreas. 2017;46(4):524-7.

13. Venkatesh PG, Navaneethan U, Vege SS. Intraductal papillary mucinous neoplasm and acute pancreatitis. J Clin Gastroenterol. 2011;45(9):755-8.

14. Rau CS, Wu SC, Chien PC, et al. Identification of Pancreatic Injury in Patients with Elevated Amylase or Lipase Level Using a Decision Tree Classifier: A Cross-Sectional Retrospective Analysis in a Level I Trauma Center. Int J Environ Res Public Health. 2018;15.pii:E277.

15. Okada R, Okada A, Okada T, Hamajima N. Elevated serum lipase levels in patients with dyspepsia of unknown cause in general practice. Med Princ Pract. 2009;18(2):130-6.

16. Pezzilli R, Morselli-Labate AM, Calculli L, Casadei R. Long-Standing Pancreatic Hyperenzymemia: Is It a Nonpathological Condition? Case Rep Gastroenterol. 2009;3(1):111-5.

17. Matsuo S, Imai E, Horio M, Yasuda Y, Tomita K, Nitta K, et al; Collaborators developing the Japanese equation for estimated GFR. Revised equations for estimated GFR from serum creatinine in Japan. Am J Kidney Dis. 2009;53(6):982-92.

18. Levin A, Stevens PE. Summary of KDIGO 2012 CKD Guideline: behind the scenes, need for guidance, and a framework for moving forward. Kidney Int. 2014;85(1):49-61.

19. Hameed AM, Lam VW, Pleass HC. Significant elevations of serum lipase not caused by pancreatitis: a systematic review. HPB (Oxford). 2015;17(2):99-112.

20. Da BL, Shulman IA, Joy Lane C, Buxbaum J. Origin, Presentation, and Clinical Course of Nonpancreatic Hyperlipasemia. Pancreas. 2016;45(6):846-9.

21. Laurent L, Vullierme MP, Rebours V, Maire F, Hentic O, Francoz C, et al. Estimation of the prevalence of intraductal papillary mucinous neoplasm of the pancreas in the French population through patients waiting for liver transplantation. United European Gastroenterol J. 2017;5(4):499-503.

22. Dorfman V, Verna EC, Poneros JM, Sethi A, Allendorf JD, Gress FG, et al. Progression of Incidental Intraductal Papillary Mucinous Neoplasms of the Pancreas in Liver Transplant Recipients. Pancreas. 2016;45(4):620-5.

23. Zhang XM, Mitchell DG, Dohke M, Holland GA, Parker L. Pancreatic cysts: depiction on single-shot fast spin-echo MR images. Radiology. 2002;223(2):547-53.

24. Pelletier AL, Hammel P, Rebours V, Couvelard A, Vullierme MP, Maire F, et al. Acute pancreatitis in patients operated on for intraductal papillary mucinous neoplasms of the pancreas: frequency, severity, and clinicopathologic correlations. Pancreas. 2010;39(5):658-61.

25. Tsutsumi K, Ohtsuka T, Oda Y, Sadakari Y, Mori Y, Aishima S, et al. A history of acute pancreatitis in intraductal papillary mucinous neoplasms of the pancreas is a potential predictive factor for malignant papillary subtype. Pancreatology. 2010;10(6):707-12.

26. Morales-Oyarvide V, Mino-Kenudson M, Ferrone CR, Gonzalez-Gonzalez LA, Warshaw AL, Lillemoe $\mathrm{KD}$, et al. Acute pancreatitis in intraductal papillary mucinous neoplasms: A common predictor of malignant intestinal subtype. Surgery. 2015;158(5):1219-25. 
27. Baiocchi GL, Portolani N, Missale G, Baronchelli C, Gheza F, Cantù M, et al. Intraductal papillary mucinous neoplasm of the pancreas (IPMN): clinico-pathological correlations and surgical indications. World J Surg Oncol. 2010;8:25.

28. Toouli J, Roberts-Thomson IC, Dent J, Lee J. Sphincter of Oddi motility disorders in patients with idiopathic recurrent pancreatitis. Br J Surg. 1985;72(11):859-63.

29. Forsmark CE. The early diagnosis of chronic pancreatitis. Clin Gastroenterol Hepatol. 2008;6(12):1291-3.

30. Bektas M, Krishna SG, Ross WA, Weston B, Katz MH, Fleming JB, et al. Prevalence of extra-pancreatic cysts in patients with cystic pancreatic lesions detected by endoscopic ultrasound. Endosc Ultrasound. 2015;4(3):219-24.

31. Byrne MF, Mitchell RM, Stiffler H, Jowell PS, Branch MS, Pappas TN, et al. Extensive investigation of patients with mild elevations of serum amylase and/or lipase is 'low yield'. Can J Gastroenterol. 2002;16(12):849-54.

32. Mortelé KJ, Wiesner W, Zou KH, Ros PR, Silverman SG. Asymptomatic nonspecific serum hyperamylasemia and hyperlipasemia: spectrum of MRCP findings and clinical implications. Abdom Imaging. 2004;29(1):109-14.

33. Pezzilli R, Morselli-Labate AM, Casadei R, Campana D, Rega D, Santini D, et al. Chronic asymptomatic pancreatic hyperenzymemia is a benign condition in only half of the cases: a prospective study. Scand J Gastroenterol. 2009;44(7):888-93.

34. Khan S, Sclabas G, Reid-Lombardo KM. Population-based epidemiology, risk factors and screening of intraductal papillary mucinous neoplasm patients. World J Gastrointest Surg. 2010;2:314-8.

\section{Tables}

Due to technical limitations, table xls is only available as a download in the Supplemental Files section.

\section{Figures}


Prevalence of IPMN and other pancreatic cystic lesions according to the serum lipase values

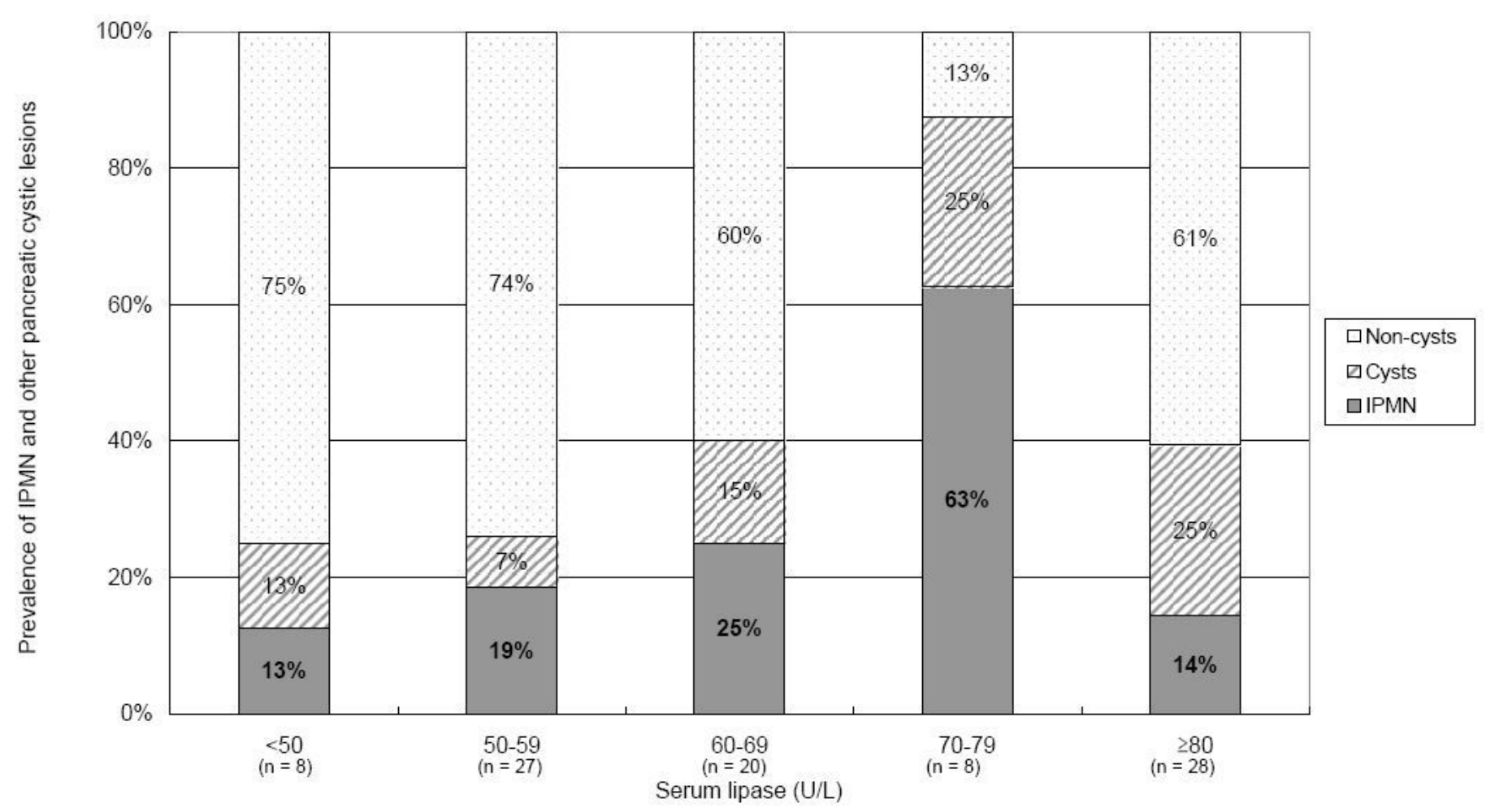

Figure 1

Prevalence of IPMN and other pancreatic cystic lesion according to the values of serum lipase. Patients who showed elevated serum lipase values (>51 U/L) during one year were included. IPMN, intraductal papillary mucinous neoplasm.

\section{Supplementary Files}

This is a list of supplementary files associated with this preprint. Click to download.

- TablesIPMN.xls 